\title{
ARTICLE
}

\section{Neutrino Properties and Their Usefulness Suggested by Recent Electrochemical Neutrino Detection Results}

\author{
Kenji ISHIBASHI $^{1 *}$, Norichika TERAO ${ }^{1}$, HAO Lijuan $^{1}$, NobuhiroSHIGYO ${ }^{1}$, and Hidehiko ARIMA ${ }^{1}$ \\ ${ }^{1}$ Kyushu University, 744, Motooka, Nishi-ku, Fukuoka 819-0395, Japan
}

\begin{abstract}
Large-scale experimental projects on neutrinos have revealed neutrino properties, particularly on a small finite neutrino mass. Alternative experiments were recently carried out with electrochemical detectors by our group. The experimental results suggest that a neutrino owns two types of potential sources and the neutrino mass generation mechanism is based on both the asymmetry of weak interactions between vector and axial-vector mass states and a scalar auxiliary field. The mass generation mechanism was formulated according to experimental indications. The asymmetry on mass functions in the axial-vector part was useful to explain the neutrino mass generation mechanism. The scalar auxiliary field was adopted which was derived from the Fermi gage. Because of the considerably large reaction rate in the electrochemical detectors, neutrinos and their fragment properties may be useful for engineering purposes in future.
\end{abstract}

KEYWORDS: neutrino, electrochemical detector, weak interaction, scalar auxiliary field, asymmetry

\section{Introduction}

Neutrinos make only weak interaction, and own a half spin of left rotation. Their detection has usually been achieved by the use of very large detector systems due to the quite small interaction cross section. Such projects as neutrino oscillation experiments have revealed that neutrinos should have a small mass in relation to the oscillation phenomena. ${ }^{1)}$

Neutrinos are treated to make the weak interaction of vector(V) and axial-vector(AV) types. ${ }^{2)}$ Electroweak theory ${ }^{3)}$ unified the electromagnetic and weak interaction, and showed that the magnitude of weak charge is completely the same as the electron charge $e$ in the electromagnetic interaction. However, such theory was not applied to the study of neutrino structure. It has recently been reported by our group that some low-energy neutrinos are readily measured with tiny electrochemical detectors. ${ }^{4,5)}$

In this paper, results of such experiments are briefly described. On the basis of matters suggested from the experimental data, the possible reason for the reaction occurrence in relation to mass-generation mechanism is attempted to be explained by assuming asymmetry of mass states between $\mathrm{V}$ and $\mathrm{AV}$ motions.

\section{Experiments by Electrochemical Detectors}

An electrochemical detector with biological material (passive detector) ${ }^{4}$ utilized a biological product raw silk and a set of electrodes in water. The output signal of the detector varied with or without nuclear-reactor neutrino irradiation. It was inferred that the raw silk produces a certain field (scalar auxiliary field, thereafter), which readily breaks a low-energy neutrino into two groups of fragments. The fragments were considered to produce the output signal. ${ }^{4)}$

Another electrochemical detector with non-biological materials required a supersonic vibration (active detector) ${ }^{5)}$

*Corresponding Author, E-mail:kisibasi@nucl.Kyushu-u.ac.jp under nuclear-reactor neutrino irradiation at an initial stage. After the initial process, the detector was sensitive to reactor neutrinos coming from nuclear power stations located in distances as long as 50-500 km level. ${ }^{5)}$ It was supposed that the scalar auxiliary field was actively produced in the initial process.

These experimental data suggest those matters as follows. A neutrino owns two types of potential sources of weakelectric charge and weak-dipole moment. The neutrino mass generation mechanism is based on the asymmetry between vector and axial-vector mass states and on the scalar auxiliary field. After interaction, neutrino fragments produce the scalar auxiliary field in turn.

\section{Neutrino Mass and Properties \\ 3.1 Assumptions}

The experimental facts induce us to make four major assumptions: (1) Interaction potentials are generated by weak charge and weak dipole moment under the Fermi gauge, (2) neutrino mass retains the property of gamma matrix ${ }^{2)} \gamma_{0}$ when the mass is described in the Dirac equation, (3) neutrino is constituted by four particles, on which four gamma matrices of $\gamma_{0}$ to $\gamma_{3}$ work as space-subspace transformation of momentum and coordinate position, and (4) asymmetry on constituent-mass properties exists between $\mathrm{V}$ and AV motions to produce the final neutrino mass. The asymmetry is applied to the potential generation (weak charge and dipole moment) in the AV part in a form of factor to the true mass.

From assumption (1), we postulate that the weak charge $Q$ has the feature of working as a weak dipole moment $Q_{d}$ in the other subspace. The dipole moment $Q_{d}$ is assumed to have a relationship between the weak charge $Q$ and specified length. It is natural to take the de Broglie wavelength $\hbar / p$ to serve as this length: $Q_{d}=Q \hbar / p$, where $p$ is the momentum of 


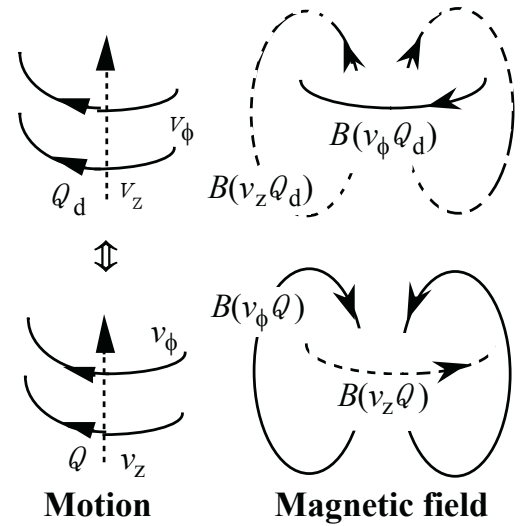

Fig. 1 Magnetic field generated by particles with electric charge $Q$ and dipole moment $Q_{d}$. The two particles are traveling in the $z$-direction with a left-rotated spiral motion.

concern. The weak dipole moment $Q_{d}$ works as the magnetic moment $\mu_{d} v Q_{d}$ for a particle moving with velocity $v$. Thereafter, $Q$ and $Q_{d}$ are called briefly charge and dipole moment, respectively. The weak- magnetic field is simply designated as magnetic one.

The basic magnetic field interaction between $Q$ and $Q_{d}$ is considered to take place as illustrated in Fig. 1. Suppose that two particles having $Q$ and $Q_{d}$ are moving in the $z$-direction with a left-rotated spiral motion. ${ }^{6}$ ) All the magnetic fields are generated in opposite directions. A simple calculation shows that magnetic fields cancel out only when neutrino velocity is close to the light one. This suggests that neutrino should basically fly with a velocity close to the light one.

\subsection{Kinetic mass formulation}

The Dirac equation for $\mathrm{V}$ and AV system is written for a constituent particle $v$ by

$$
\sum_{\mu=0 \sim 3}\left(\gamma^{\mu} p_{v V}^{\mu}+\gamma^{5} \gamma^{\mu} p_{v A}^{\mu}\right) \psi_{v}=m_{v} \psi_{v},
$$

where potentials are neglected for simplicity, and symbols are defined as

$$
\begin{aligned}
& \gamma^{\mu}=\gamma_{\mu} \text { for } \mu=0 \sim 3, \quad \gamma^{5}=\gamma_{5} / i, \\
& p_{v V}^{\mu}=p_{\mu, V V}, \quad p_{v A}^{\mu}=i p_{\mu, v A} .
\end{aligned}
$$

From this definition, the complex momentum $p_{v V}^{\mu}$ of V-type takes a real value while that of AV-type $p_{v A}^{\mu}$ has an imaginary one. According to assumption (2), we replace $m_{v}$ in eq. (1) by $\gamma^{0} m_{v}$. This replacement is useful for describing potential generation by reversed-type complex motion ${ }^{6)}$ in relation to assumption (4). Further multiplication of eq. (1) with $\gamma^{0} m_{v}$ by $\gamma^{0}$ leads to

$$
\sum_{\mu=0 \sim 3}\left(\gamma^{0} \gamma^{\mu} p_{v V}^{\mu}+\gamma^{0} \gamma^{5} \gamma^{\mu} p_{v A}^{\mu}\right) \psi_{\nu}=m_{\nu} \psi_{\nu} .
$$

This equation is considered to govern the motion of constituent particles. Since there are four principal gamma matrices $\gamma_{0}$ to $\gamma_{3}$, we consider that four constituent particles take part in the neutrino internal motion. These constituents are designated as particle $v=0$ to 3 .
The eigenvalue of mass is derived from eq. (2) with Pauli's spin matrices $\sigma_{0}$ to $\sigma_{3}$ as

$$
m_{v}=\left(p_{v V}^{0}-h p_{v A}^{0}\right) \sigma_{0}+\sum_{k}\left(p_{v V}^{k}-h p_{v A}^{k}\right) \sigma_{k},
$$

where we take the parameter $h=-1$ as a meaningful solution. The squared value leads to

$$
\begin{aligned}
\left(m_{v}\right)^{2}=\left(p_{v V}^{0}\right. & \left.-h p_{v A}^{0}\right)^{2}+\sum_{k}\left(p_{v V}^{k}-h p_{v A}^{k}\right)^{2} \\
& +2\left(p_{v V}^{0}-h p_{v A}^{0}\right) \sum_{k}\left(p_{v V}^{k}-h p_{v A}^{k}\right) \sigma_{k} .
\end{aligned}
$$

Finally, the squared mass should become scalar. Therefore, we impose the constraint of

$$
p_{v V}^{0}-h p_{v A}^{0}=0 .
$$

On the basis of assumption (3), we consider the gamma matrixes have a role of transformation of momentum from a usual conventional space $U^{c}$ to individual subspace $U^{v}$, where the constitution particles possibly exist in most cases. The transformation matrix is expressed as

$$
U_{v}=\gamma_{v} \text { for } v=0,1,3 \text {, and } U_{2}=\gamma_{2} / i \text {. }
$$

Imaginary elements in the transformation matrices are regarded as the conversion into (quasi) imaginary-mass state. We impose that the squared value of mass in eq. (4) should be scalar in $U^{v}$. The momentum after conversion is indicated by dashed mark. We have an additional constraint of

$$
p^{\prime 0}-h p^{\prime 0}{ }_{v A}^{0}=0
$$

in $U^{v}$. If this is expressed with the values in the original space $U^{c}$, eq. (6) becomes to

$$
p_{v V}^{\kappa_{V}}-h p_{v A}^{\kappa_{v}}=0,
$$

with $\kappa_{v}=0,3,3,2$ for particles $v=0,1,2,3$, respectively. The root of squared mass in eq. (4) leads to the kinetic mass

$$
m_{v}= \pm \sqrt{\sum_{\mu}\left(p_{v V}^{\mu}\right)^{2}+\sum_{\mu}\left(p_{v A}^{\mu}\right)^{2}}
$$

where constraints of eqs. (5) and (7) are applied to momentums. Since the AV momentums are imaginary in eqs. (5) and (7), they disagree with those of V. We admit the AV motions in eqs. (5) and (7) to behave with reversed complex type, that is, they work with apparent imaginary mass of $i m_{v}$. This corresponds to apparent change of mass property in AV motion according to assumption (4). The value of $m_{v}$ in eq. (8) takes either plus or minus, which is called positive or negative mass state, respectively.

The Dirac equation describes the motion of half-integer spin particles. The wave function in eq. (2) should express the combination motion of these particles. The situation is explained in Fig. 2. Since particle 1 is hard to reside in $U^{1}$ due to the conversion property of $U_{1}$, it is supposed to always work in $U^{c}$. Particle 1 is considered to offer position bases to other particles, and take the charge-type potential source of $Q_{1}$ in $U^{c}$. In contrast, particle $v$ basically has the dipole moment $Q_{d v}$ in subspace $U^{v}$. Because of sharing of $x_{v b}{ }^{\mu}$, the motions of $x_{1}{ }^{\mu}$ and $x_{v}{ }^{\mu}$ are present in close positions 


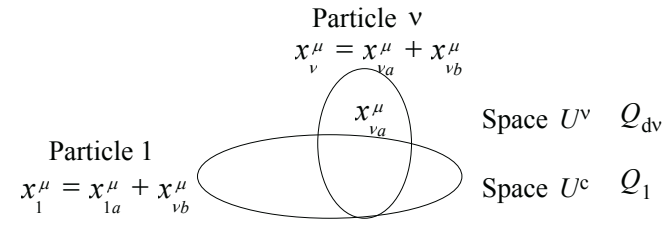

Fig. 2 Illustration of positional structure for particle motion. Particle 1 exists in the space $U^{c}$, offering position base to another particle $v$.

to keep the situation of magnetic field cancelling in Fig. 1. The canonical conjugate momentum in the wave function in eq. (2) applies to the motion of combined positions such as $x_{1}{ }^{\mu}$ and $x_{v}^{\mu}$.

For particle 1, the position $x_{1 a}{ }^{\mu}$ makes an actual motion, while the whole motion of $x_{1}{ }^{\mu}$ formally constitutes the canonical conjugate momentum. However, it is useful to consider a secondary wave equation to describe the motion of $x_{1 a}{ }^{\mu}$. The equation may be a Klein-Gordon type one with second-order derivatives due to more extent of freedom. Meanwhile, we admit particle 1 to have an internal negative mass state of 1 - in addition to positive one of $1+$. In fact, one of particles among 0,2 and 3 may has an opposite mass polarity to others, in order to achieve a resultant small mass. The internal negative mass state 1 - having the negative charge is required to magnetically couple with a particle of negative mass owning a negative dipole moment $Q_{d v}$. The second order equation for $x_{1 a}{ }^{\mu}$ motion is thus written by

$$
\begin{gathered}
\sum_{\pi= \pm} s_{1}^{\pi} \sum_{\mu=0 \sim 3}\left\{\left(\gamma^{0} \gamma^{\mu}\right)^{2}\left(p_{1 a V \pi}^{\mu}\right)^{2}+\left(\gamma^{0} \gamma^{5} \gamma^{\mu}\right)^{2}\left(p_{1 a A \pi}^{\mu}\right)^{2}\right\} \psi_{1 a} \\
=\left(m_{1}\right)^{2} \psi_{1 a}, \\
m_{1}=\sqrt{\sum_{\pi= \pm} s_{1 a}^{\pi} \sum_{\mu=0 \sim 3}\left\{\left(p_{1 a V \pi}^{\mu}\right)^{2}+\left(p_{1 a A \pi}^{\mu}\right)^{2}\right\}}, \\
s_{1}^{ \pm}= \pm 1, \quad \psi_{1 a}=\psi_{1 a+} \psi_{1 a-} .
\end{gathered}
$$

\subsection{Total Kinetic Mass and Total Energy}

Total kinetic mass $m$ of neutrino is given by a linear sum of individual masses with polarity $s_{v}^{m}$ as

Table 1 Allocation of angular momentums in $U^{c}$. The spin part $l_{s}$ is shared by orbital motions of particles 0,2 and 3 . Symbols for linear combination is listed below.

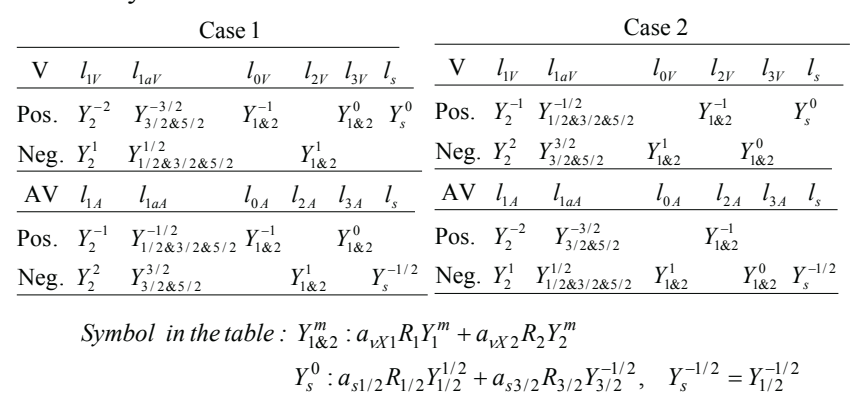

$$
\begin{aligned}
& m=\sum_{v} m_{v}=\sqrt{m \sum_{v} s_{v}^{m}\left|m_{v}\right|}=\sqrt{\sum_{v} s_{v}^{m}\left|m_{v}^{\text {int }}\right|^{2}} \\
&=\sqrt{\sum_{\pi= \pm} s_{1}^{\pi}\left\{\sum_{\mu=0 \sim 3, X=V, A}\left(m / m_{1}\right)\left(p_{1 X \pi}^{\mu}\right)^{2}\right\}} \\
&+\sum_{v=0,2,3}\left\{\sum_{\mu=0 \sim 3, X=V, A}\left(m / m_{v}\right)\left(p_{v X}^{\mu}\right)^{2}\right\}
\end{aligned}
$$

The value of $m_{v}{ }^{i n t}$ indicates the intrinsic internal mass, while $m_{v}$ is interpreted as an effective mass in a view of external motion. The total neutrino energy $E$ is expressed by

$$
E=\sqrt{\sum_{\pi= \pm}\left\{\sum_{\mu=0 \sim 3, X=V, A}\left(m / m_{1}\right)\left(p_{\mu, 1 X \pi}\right)^{2}\right\}} .
$$

\subsection{Total spin}

The total $z$-direction and squared-total angular momentum operators $L^{z}$ and $(L)^{2}$ are defined as eigenvalues for $\mathrm{V}$ and $\mathrm{AV}$ angular momentum sum in matrices. The operators $L^{z}$ and $(L)^{2}$ and those of sum of right-hand side on particles $v=0,2,3$ in eq. (3) should be exchangeable in a commutator operation. The commutator between $L^{z}$ and mass operators gives an eigenvalue of $L_{z}=-1 / 2$ and the corresponding eigenvector state of

$$
\sum_{\substack{v=0 \sim 3 \\ X=V, A}}\left\{\sin \theta_{v X} p_{v X}^{r}\right\}=\sum_{\substack{v=0 \sim 3 \\ X=V, A}}\left\{\left(i / r_{v X} \sin \theta_{v X}\right) l_{v X}^{\phi}\right\} .
$$

Since $l_{v X}{ }^{\phi}$ gives a fixed value, the total value in right-hand-side gives a constraint to the radial momentum in the left-hand-side. Equation (13) forbids the state of all $p_{v X}{ }^{r}=0$ due to the non-zero sum in the right-hand side. It is considered that the radial motions appear in either expanding or shrinking state. When the periodical radial-velocity polarity change is always followed by a simultaneous mass polarity change, $p_{v X}{ }^{r}$ is kept in a continuous value during the transition. For this reason, there are two cases, where the radical velocity is either positive (case 1) or negative (case 2). The view leads to periodical vibration of radial motion, with keeping canonical conjugate radial momentum at the transition.

\subsection{Spherical Functions with Linear Combination}

The candidates of angular wave functions are listed in Table 1 with symbolized forms. The wave functions are basically expressed by a linear combination to two functions, for example,

$$
Y_{1 \& 2}^{m}: \psi=\cos \Theta R_{1} Y_{1}^{m}+\sin \Theta R_{2} Y_{2}^{m},
$$

where the mixing angle $\Theta$ constitutes linear coefficients. Since an expected value of $z$ varies with $\Theta$, the mixing angle determines the particle form variation in the $z$ direction. If difference between $\Theta_{v V}$ and $\Theta_{v A}$ for particles $v=0,2,3$ are set 
Table 2 Potential sources with asymmetry in AV2.

\begin{tabular}{c||c|cc}
\hline & $\mathrm{V}$ & AV1 & AV2 \\
\hline \hline Particle 0 & $Q_{d 0}$ & $Q_{d 0}$ & $i Q_{d 0}$ \\
\hline Particle 2 & $Q_{d 2}$ & $Q_{d 2}$ & $-Q_{2}$ \\
\hline Particle 3 & $Q_{d 3}$ & $Q_{d 3}$ & $i Q_{d 3}$ \\
\hline
\end{tabular}

at $\pi / 2$, the sum of orbital angular momentums on particles $0,2,3$ leads to the state of $Y_{2}^{0}$ under anti-symmetrization of wave functions between $\mathrm{V}$ and $\mathrm{AV}$ types. When particle 1 makes four wave functions of $Y_{2}^{ \pm 1}$ and $Y_{2}^{ \pm 2}$, their summation with the state $Y_{2}^{0}$ leads to $Y_{0}^{2}$. The orbital angular momentums, thus, cancel out in neutrino, and subsequently the spin part of $-1 / 2$ remains in the total angular momentum.

\subsection{Asymmetry}

When potentials ${ }^{6}$ are included, the momentums in eqs. (8), (10) and (12) are replaced in such a way as

$$
p_{v X}^{k} \rightarrow p_{v X}^{k}-Q_{d v} B_{v X}^{k} \quad \text { or } \quad p_{1 a X \pi}^{k} \rightarrow p_{1 a X \pi}^{k}-\pi Q_{1} A_{1 a X \pi}^{k} .
$$

It is straight-forward to show that masses of eqs. (8) and (10) become zero after anti-symmetrization of wave functions between $\mathrm{V}$ and AV types. Symmetry breaking in mass property between $\mathrm{V}$ and $\mathrm{AV}$ types will generate the mass values through potential difference between $\mathrm{V}$ and AV. The previous asymmetry in section 3.2 was the setting of the reversed complex type momentum in AV motion. The additional asymmetry is imposed as follows. According to assumption (4), we consider that the AV motion state of particles 0,2,3 is divided into two: AV1 and AV2 with the same half probability. The asymmetry is set at AV2 for potential interaction. The AV1 makes the usual potential interaction based on $Q_{d v}$ coming from the mass $m_{v}$. The AV2 is assigned to $x_{v b}{ }^{\mu}$ motion in Fig. 2, and admitted to work with factor $e^{i \zeta_{v}}$ as

$$
Q_{d v} e^{i \zeta_{v}} \text { or } Q_{v} e^{i \zeta_{v}} \text {, corresponding to } m_{v} e^{i \zeta_{v}} \text {. }
$$

The situation of this asymmetry is summarized in Table 2. The asymmetry creates the difference in $\mathrm{V}$ and $\mathrm{AV}$ potentials through generation of the scalar auxiliary field $B_{A}{ }^{0}$, which appear as $\left(Q_{d v} B_{A}{ }^{0}\right)^{2}$ in the root of eqs. (8), (10) and (12).

\subsection{Calculation Example and Discussion}

The calculation example is shown in Table 3 for neutrino with energy of $1 \mathrm{MeV}$. The kinetic mass $m_{v}$ came from eqs. (8) and (10), while the self mass ${ }^{6)}$ was obtained from the self electromagnetic energy at the particle position. The calculation utilized an approximation with rotationally symmetric potentials around $\mathrm{z}$-axis, and gives the neutrino mass in meV level in the observation frame. The usual neutrino has a velocity close to the light one. If a low-energy neutrino receives ${ }^{4)}$ an external scalar auxiliary field $B_{A}{ }^{0}$ producing an interaction energy of $\mathrm{meV}$ to $\mathrm{eV}$ level, the field increases the kinetic neutrinos mass. When the neutrino velocity becomes below threshold energy, it is impossible to
Table 3 Calculation example of constituent masses $(\mathrm{eV})$ for neutrino of $1 \mathrm{MeV}$. Sum(obs) indicates the total mass that is seen by observers.

\begin{tabular}{c||cccc||cc}
\hline Part. No. & 0 & 1 & 2 & 3 & Sum & Sum(obs) \\
\hline \hline Case 1 & 365 & 154 & -367 & 2.0 & 154 & $2.4 \times 10^{-2}$ \\
\hline Case 2 & -347 & 139 & 349 & -1.8 & 139 & $1.9 \times 10^{-2}$ \\
\hline
\end{tabular}

take the magnetic configuration between $Q$ and $Q_{d}$ motions. The interaction between $Q$ and $Q_{d}$ is considered to be somewhat repulsive at rest due to the magnetic interaction. Then, the neutrino may break up two groups ${ }^{4}$ of fragments: particle 1 of $Q$ type and a sum of particles $0,2,3$ with $Q_{d}$. The particles 0,2,3 lose the sharing position of $x_{v b}$. Experimental data suggest ${ }^{4}$ that they should be attached to an electron to seek such a sharing function, while particle 1 tends to be combined with a proton.

\section{Conclusion}

The experimental results by recent electrochemical detectors suggest that a neutrino owns two types of potential sources of weak charge and weak dipole moment, and the neutrino mass generation mechanism is based on asymmetry of mass factors and on the scalar auxiliary field. The mass generation mechanism was formulated on the basis of experimental indications. The scalar auxiliary field was derived with the Fermi gage. Assumption of asymmetry on mass working in the AV-part was useful to explain the neutrino mass generation. It was inferred that the application of external AV-type scalar auxiliary field may bring about neutrino break up into fragments.

\section{References}

1) Q. R. Ahmad, et al., "Direct Evidence for Neutrino Flavor Transformation from in Sudbury Neutrino Observatory," Phys. Rev. Letters, 89, 011301 (2002).

2) J. D. Bjorken, S. D. Drell, Relativistic Quantum Fields, McGraw-Hill (1965).

3) S.L. Glashow, "The status of the electroweak sector of the standard model," Nucl. Phys. 22, 579-588 (1961), A. Salam, "Weak and Electromagnetic Interactions," Elementary Particle Theory (ed. By N. Svartholom, Almquist and Forlag. 1968), 367-377, S. Weinberg, “A Model of Leptons," Phys. Rev. Letters, 19, 1264-1266 (1967).

4) K.Ishibashi, Liu Wei, et al., "Electrochemical Neutrino Detector and Its Signal Generation Scenario in Relation to Oxygen Generation," presented at this symposium.

5) N.Terao, K.Ishibashi, et al., "Attempt at Neutrino Detection by Resonance-Type Electrochemical Detector with a Small Size," J. Nucl. Sci. and Techonol. Suppl.5, 670-673 (2008).

6) Hao Lijuan, K.Ishibashi, et al., "Proposed Weak Potential Generation in Creating Self-Mass of Neutrinos," presented at this symposium. 